\title{
Potentials of spectroscopic studies of comets and exoplanets with WSO-UV mission
}

\author{
E. Kanev, S. Sichevsky, V.Shmagin and M.Sachkov \\ Institute of Astronomy, \\ Russian Academy of Sciences(E-mail: kanev@inasan.ru)
}

Received: August 5, 2021; Accepted: November 28, 2021

\begin{abstract}
In this article we consider the prospects of research on comets and exoplanets using instruments onboard the WSO-UV mission. Ultraviolet spectroscopy at 115-310 $\mathrm{nm}$ is a powerful tool for studying comets, since this range of the electromagnetic spectrum contains most of the resonance lines of atoms, molecules and ions. The spectrographs on board the WSO-UV spacecraft will become one of the most important instruments for spectral studies of comets and exoplanets after 2025 .
\end{abstract}

Key words: UV - comets - exoplanets

\section{Introduction}

Observations in the ultraviolet (UV) range of the electromagnetic spectrum are the key to solving many problems in determining the chemical composition of comets. UV spectroscopy provides important information about the composition of a cometary coma. For example, radiation elements that originate from the products of dissociation of water: $\mathrm{OH}, \mathrm{H}$ and $\mathrm{O}$, or correspond to secondary atomic, molecular and ionic forms, such as $\mathrm{C}, \mathrm{C}+, \mathrm{CO}, \mathrm{CO}+, \mathrm{CO}+2$ dominate in a cometary coma. Spectra and images provide information about the structure and dynamic characteristics of the coma and help to reveal the structure and other properties of the nuclei.

Comets are difficult to observe. We are limited to observing at a distance, so the key task is to distinguish between the signatures of the coma and the nucleus. Another problem is N2 and noble gases, which are very volatile and evaporate at very low temperatures. Thus, they are of particular interest for the thermal history of comets. But their UV-signature in a cometary coma has yet to be found. Observations of comets from space have several specific features (Sachkov, 2016). One of the space projects fitting most of the requirements for cometary observations is the World Space Observatory - Ultraviolet (WSO-UV). WSO-UV is a multipurpose international space mission created in response to the growing demand for UV facilities by the astronomical community (Sachkov et al., 2018). 


\section{Scientific tasks}

The main scientific tasks of the WSO-UV observatory consist in spectroscopy of faint sources and imaging in the UV part of the spectrum. At the same time, the following should be achieved: high spectral resolution, maximum spatial resolution, and, for studies of faint sources, high permeability. The choice of parameters of the T-170M telescope (large diameter of the primary mirror (170 $\mathrm{cm})$, special coatings that improve reflection in the UV range, high-precision guidance and stabilization systems, etc.) is due to the necessity for maximum angular resolution and maximum effective area in the range of 110-320 $\mathrm{nm}$, in order to provide high-resolution spectroscopy and high-quality imaging (Sachkov et al., 2019).

\subsection{Comets in UV with WSO-UV}

A number of problems in the field of cometary research, in particular, the determination of the chemical composition of comets and the study of physical processes in their nuclei, can be solved only with the use of observational data in the UV range of the electromagnetic spectrum. Due to the opacity of the Earth's atmosphere, such studies can only be carried out by methods of exoatmospheric astronomy. At the same time, exoatmospheric cometary observations have a number of peculiarities compared to such studies of other astronomical objects.

Let's list the most significant of them. Comets are moving objects. Their speed can reach tens of arcseconds per hour. For example, comet Halley moves at 11" per hour relative to the stars. To obtain UV spectra, it is necessary not only to adjust the stabilization of the spacecraft during long exposures, but also to have a sufficiently accurate a priori knowledge of this velocity. It is important, especially for spectral studies, to take into account the dependence of the relative intensity of solar and cometary lines in the spectrum on the comet velocity. This is the so-called swing effect. It is associated with the fact that the lines in the solar radiation spectrum are shifted by the magnitude of the comets heliocentric velocity. The presence of solar lines in the spectrum significantly complicates observations for some cometary velocities. For example, for the C I $165 \mathrm{~nm}$ line at a speed of more than $40 \mathrm{~km} / \mathrm{s}$, the cometary line will be recorded three times weaker than the maximum possible.

Another important aspect of UV spectral studies of comets is the fact that comets are variable objects. Cometary nuclei produce short-lived tails and comas, which require continuous or quasi-continuous observations. Comets are extended objects. The most suitable instrument for their spectral research is a high-slit (long-slit) spectrograph. Such spectrographs were used in the rocket missions for observation of comet Hale-Bopp in 1997. 


\subsection{Exoplanets in UV with WSO-UV}

Given the large aperture and the instrument sensitivity, WSO-UV will be one of the major workhorse for exoplanet observations. The current exoplanet research can be divided into five major aspects:

- planet detection,

- planet formation and migration,

- planets physical properties,

- planet's chemical properties and biomarkers,

- planets as a tool to study the properties of the host stars.

The WSO-UV mission will be a crucial tool for the study of the last four aspects (Fossati et al., 2014).

\section{WUVS instruments of the WSO-UV mission}

The spectroscopic capabilities of WSO-UV and HST are rather similar; in the NUV range, WSO-UV spectrographs will be more efficient than the HST/STIS spectrograph however, the sensitivity of HST/COS in the FUV range will not be matched by WSO-UV:

- high-resolution spectroscopic observations of pointlike objects in the 115 $320 \mathrm{~nm}$ spectral range. Resolving power of the two high resolution spectrographs designed for observations in the NUV $(180-320 \mathrm{~nm})$ and FUV (110 $-180 \mathrm{~nm})$ is $R>50000$;

- low-resolution (resolving power $R>1000$ ) spectral observations. A long slit spectrograph will operate in the $115-320 \mathrm{~nm}$ range too. It is especially suitable for faint and extended objects such as comets.

\section{UV SPectrographs of the exospheres of Earth-like EXo- planet (UVSPEX)}

In cooperation Russia-Japan FCU will be equipped UVSPEX spectrograph with MCP detector in the photon counting mode records spectra in the range of 115 - $135 \mathrm{~nm}$. It is expected the spectrograph has a spectral resolution better than $0.5 \mathrm{~nm}$. Thus, bservations of oxygen line OI (triplet $130.2-130.6 \mathrm{~nm}$ ) during exoplanet transit can distinguish an Earth from a Venus or Mars (Tavrov et al., 2018). 


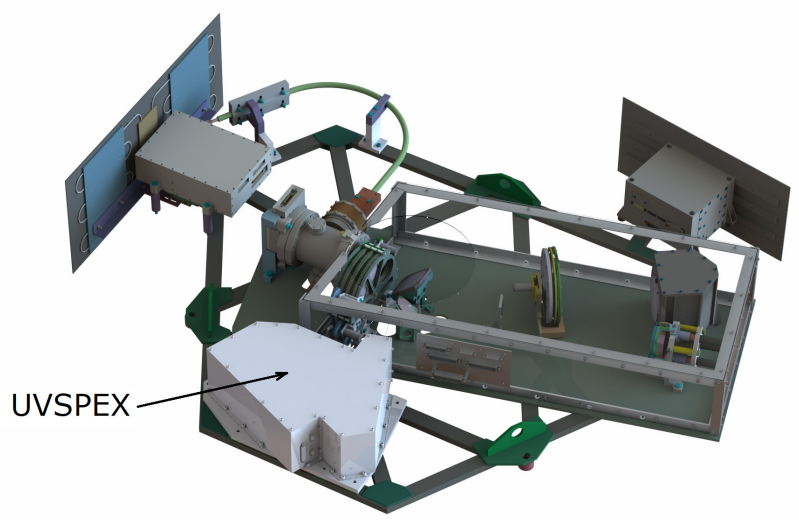

Figure 1. FCU general view.

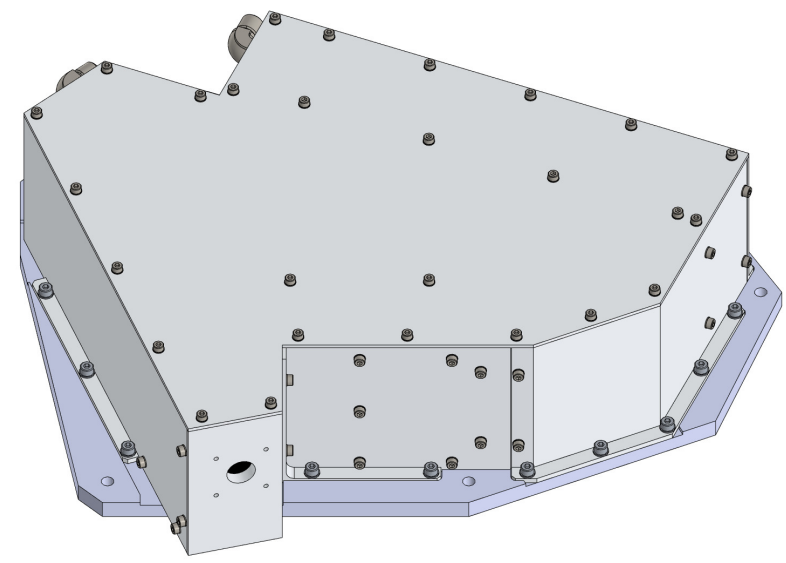

Figure 2. UVSPEX general view.

\section{5. conclusions}

The WSO-UV space observatory, which will be equipped with low- and highresolution spectrographs, will be a very significant tool for cometary studies. Due to its high-resolution spectrographs and UVSPEX instrument, it will also become one of the main workhorses for exoplanet observations. WSO-UV will be ohe of the most important tool for the spectral studies of comets and exoplanets after 2025 . 
Acknowledgements. Authors acknowledge the support of Ministry of Science and Higher Education of the Russian Federation under the grant 075-15-2020-780 (N13.1902.21.0039).

\section{References}

Fossati, L., Bisikalo, D., Lammer, H., Shustov, B., \& Sachkov, M., Major prospects of exoplanet astronomy with the World Space Observatory-UltraViolet mission. 2014, Astrophysics and Space Science, 354, 9, DOI: 10.1007/s10509-014-2027-3

Sachkov, M., Panchuk, V., Klochkova, V., et al., Spectroscopic instrumentation of 1-m class telescopes for ground support of the space mission WSO-UV. 2019, Contributions of the Astronomical Observatory Skalnate Pleso, 49, 142

Sachkov, M. E., Spectral studies of comets in the ultraviolet range and prospects of the WSO-UV project in these studies. 2016, Solar System Research, 50, 294, DOI: $10.1134 /$ S0038094616040055

Sachkov, M. E., Kartashova, A. P., \& Emel'yanenko, V. V., Prospects for spectral studies of comets: The World Space Observatory - Ultraviolet project. 2018, Planetary Space Science, 164, 75, DOI: 10.1016/j.pss.2018.06.013

Tavrov, A., Kameda, S., Yudaev, A., et al., Stellar imaging coronagraph and exoplanet coronal spectrometer: two additional instruments for exoplanet exploration onboard the WSO-UV 1.7-m orbital telescope. 2018, Journal of Astronomical Telescopes, Instruments, and Systems, 4, 1, DOI: 10.1117/1.JATIS.4.4.044001 